\title{
The dynamics of miscible viscous fingering from onset to shutdown
}

\author{
Japinder S. Nijjer ${ }^{1} \dagger$, Duncan R. Hewitt ${ }^{1}$, and Jerome A. Neufeld ${ }^{1,2,3}$ \\ ${ }^{1}$ Department of Applied Mathematics and Theoretical Physics, University of Cambridge, \\ Wilberforce Road, Cambridge CB3 0WA, UK \\ ${ }^{2}$ Department of Earth Sciences, University of Cambridge, Downing Street, Cambridge CB2 \\ 3EQ, UK \\ ${ }^{3}$ BP Institute, University of Cambridge, Bullard Laboratories, Madingley Road, Cambridge \\ CB3 0EZ, UK
}

(Received xx; revised xx; accepted xx)

We examine the full "life cycle" of miscible viscous fingering from onset to shutdown with the aid of high-resolution numerical simulations. We study the injection of one fluid into a planar two-dimensional porous medium containing another, more viscous, fluid. We find that the dynamics are distinguished by three regimes: an early-time linearly unstable regime, an intermediate-time non-linear regime, and a late-time single-finger exchangeflow regime. In the first regime, the flow can be linearly unstable to perturbations that grow exponentially. We identify, using linear stability theory and numerical simulations, a critical Peclet number below which the flow remains stable for all times. In the second regime, the flow is dominated by the non-linear coalescence of fingers which form a mixing zone in which we observe that the convective mixing rate, characterized by a convective Nusselt number, exhibits power-law growth. In this second regime we derive a model for the transversely averaged concentration which shows good agreement with our numerical experiments and extends previous empirical models. Finally, we identify a new final exchange-flow regime in which a pair of counter-propagating diffusive fingers slow exponentially. We derive an analytic solution for this single-finger state which agrees well with numerical simulations. We demonstrate that the flow always evolves to this regime, irrespective of the viscosity ratio and Peclet number, in contrast to previous suggestions.

\section{Introduction}

Mixing of fluids in porous media is notoriously difficult due to the absence of inertia, and lies at the heart of many real-world problems: it plays a key role in carbon sequestration (Huppert \& Neufeld 2014), oil recovery (Lake 1989), mantle convection (van Keken et al. 2002), microfluidic devices (Stone et al. 2004) and food processing (Hill 1952). Ultimately mixing occurs as molecular diffusion acts to reduce local concentration gradients. It is, therefore, most effective when both gradients in concentration, and the surface areas across which it acts, are large. While fluids at high Reynolds numbers can be vigorously stirred by turbulence, other mechanisms are required to stir fluids in a porous medium. One such mechanism is the generation of interfacial instabilities, which increase the area over which molecular diffusion acts. Such instabilities can be driven by a number of different mechanisms including chemical reactions (Almarcha et al. 2010), unstable density stratifications (Hewitt et al. 2013) and differences in viscosity (Tan \& Homsy 1988). Here we focus on the effects of a planar, viscously unstable interface on mixing in porous media.

$\dagger$ Email address for correspondence: jn350@damtp.cam.ac.uk 
Viscous fingering is an interfacial instability that occurs when a less-viscous fluid displaces a more-viscous one in a porous medium or Hele-Shaw cell. This phenomenon was first described by Hill (1952) and later by Saffman \& Taylor (1958). The instability results in a series of fine fingers whose length scale can depend on a variety of factors including surface tension and diffusion. Saffman and Taylor showed that in the case of immiscible flows (when the fluids do not mix) these fingers tend to coalesce to a single steadily-propagating finger. Since the work of Saffman and Taylor, there have been a variety of studies on both the initial instability and the stability of the single-finger state (see McCloud \& Maher 1995).

If the interfacial tension is zero, Saffman and Taylor's theory predicts infinitesimally small fingers. However, experiments with miscible fluids indicate that diffusion between the fluids acts to smear the smaller scales leading to finite wavelength fingers (Lajeunesse et al. 1999; Bischofberger et al. 2014). Tan \& Homsy (1986) used linear stability theory, and a slowly diffusing background flow, to predict the most unstable mode and its growth. In subsequent work, they compared their theory to numerical simulations of the full $2 \mathrm{D}$ problem (Tan \& Homsy 1988). Since then much work has been done to understand the onset and early-time behaviour with the inclusion of a variety of stabilizing and destabilizing mechanisms such as gravity (Ruith \& Meiburg 2000), Korteweg stresses (Pramanik \& Mishra 2015a,b) and permeability layering (De Wit \& Homsy 1997a,b).

Some recent attempts have been made to model the impact of viscous fingering on mixing beyond the onset. Jha et al. $(2011 a, b)$ examined the long-time mixing of a viscously unstable system containing high or low viscosity blobs in a doubly periodic domain. Informed by numerical experiments, they developed a model for the evolution of the mixing rate. Here, we instead investigate the evolution of a single viscously unstable planar interface from onset to shutdown.

Although previous work has looked at the onset problem and early-time behaviour of miscible viscous fingering, the late-time behaviour remains poorly understood. In previous work, Tan \& Homsy (1988) determined a critical Peclet number beyond which tip-splitting occurs, and they hypothesized that this value might have implications for the asymptotic fate of the fingers. Zimmerman \& Homsy (1992) similarly suggested that the asymptotic behaviour may include multiple steadily propagating fingers under the assumption that tip splitting may balance the upwards cascade in the scale of the fingers, but were unable to extend their numerical simulations to a final state. In experiments in a radial geometry, Chui et al. (2015) showed a transition in finger growth from a scaling with $t$ to one with $t^{\frac{1}{2}}$ corresponding to the shutdown of the instability. However, the ultimate fate and final form of the fingers remains unclear.

This paper has two main aims. The first aim is to identify and provide a detailed explanation of the asymptotic fate of the fingering instability. Then, given an understanding of the late-time behaviour, the second aim is to examine the full "life cycle" of miscible viscous fingering from "onset" to "shutdown" which draws together previously disjoint or contradictory observations and claims. We find that the dynamics can be divided into three regimes: (i) at early times, the flow is well-described by linear stability theory; (ii) at intermediate times, the flow is dominated by non-linear finger interactions; and (iii) at late times, the flow is composed of exponentially slowing single-finger exchange-flow. Ultimately, once the fingers have slowed enough, diffusion in the direction of the flow dominates the dynamics. In the course of this study, we also identify a critical Peclet number for the instability in the first regime and derive an improved averaged model for the flow in the second regime.

This paper is laid out as follows. In $\S 2$ we formulate the problem, and we describe 


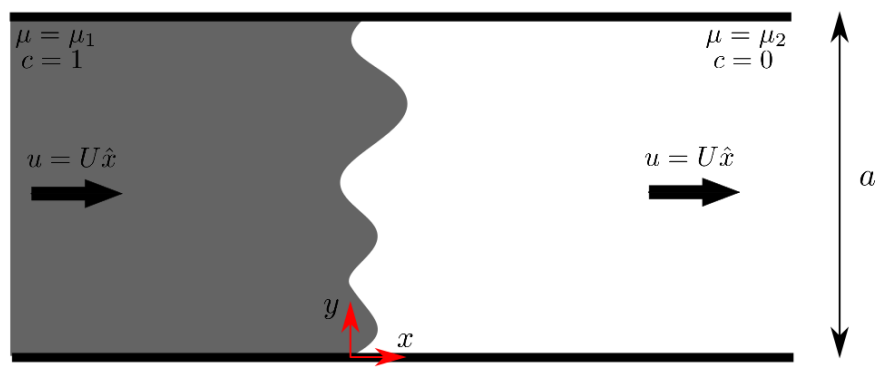

Figure 1. An illustration of the model setup. The porous medium is taken to be an infinite strip of width $a$ initially filled with a fluid with viscosity $\mu_{2}$. A fluid with viscosity $\mu_{1}$ is injected at a constant velocity $U \hat{\boldsymbol{x}}$ into the medium. We measure the concentration of the injected fluid, which is one upstream and zero downstream.

the numerical method used to solve this problem in $\S 3$. In $\S 4$ we present numerical results across a range of parameter settings and identify the dominant scalings in each regime. We then discuss the early-time linearly unstable and intermediate-time non-linear coalescence regimes in more detail in $\S 5$. Finally, in $\S 6$, we discuss the late-time behaviour, for which we derive an analytic solution for the new single-finger state and compare it to the results of our numerical simulations.

\section{Problem formulation}

We consider a two-dimensional, isotropic porous strip of infinite extent and finite width $a$ (figure 1). The medium has uniform porosity $\phi$ and permeability $k$, and is initially saturated with an ambient fluid which has viscosity $\mu_{2}$. Another fluid, which is fully miscible with the ambient fluid and has viscosity $\mu_{1}$, is injected at a constant velocity $U \hat{\boldsymbol{x}}$. The diffusivity between the fluids is $D$ and gravity is neglected. Note that, in general, the permeability and diffusivity may be described by second-rank tensors, and can depend on a variety of factors including the concentration of either fluid, fluid velocity, time, and space. For simplicity, the permeability and diffusion-dispersion tensors are here assumed to be isotropic and constant.

\subsection{Governing equations}

The two fluids are incompressible and fully miscible. The flow obeys Darcy's law and the concentration of the injected fluid is described by an advection-diffusion equation,

$$
\begin{gathered}
\nabla \cdot \mathbf{u}=0, \\
\mathbf{u}=-\frac{k}{\mu(c)} \nabla p, \\
\phi \frac{\partial c}{\partial t}+\mathbf{u} \cdot \nabla c=\phi D \nabla^{2} c .
\end{gathered}
$$

Here $\mathbf{u}=(u, v)$ is the Darcy velocity or fluid flux, $p$ the pressure, and $c$ the concentration, which varies between 0 (in the ambient fluid) and 1 (in the injected fluid). The viscosity 
$\mu(c)$ varies with the concentration, and we follow the convention of previous authors (e.g. Tan \& Homsy 1986; Zimmerman \& Homsy 1991; Pramanik \& Mishra 2015a) by assuming an Arrhenius-like exponential dependence,

$$
\mu(c)=\mu_{2} e^{-R c},
$$

where $R=-\ln \left(\mu_{1} / \mu_{2}\right)$.

We non-dimensionalize the equations by the height of the domain $a$, velocity $U$, time $\phi a / U$, permeability $k$, viscosity of the ambient fluid $\mu_{2}$, and pressure $\mu_{2} U a / k$, leading to

$$
\begin{gathered}
\frac{\partial u^{*}}{\partial x^{*}}+\frac{\partial v^{*}}{\partial y^{*}}=0, \\
-u^{*} \mu^{*}=\frac{\partial p^{*}}{\partial x^{*}}, \quad-v^{*} \mu^{*}=\frac{\partial p^{*}}{\partial y^{*}}, \\
\frac{\partial c}{\partial t^{*}}+u^{*} \frac{\partial c}{\partial x^{*}}+v^{*} \frac{\partial c}{\partial y^{*}}=\frac{1}{\operatorname{Pe}}\left(\frac{\partial^{2} c}{\partial x^{* 2}}+\frac{\partial^{2} c}{\partial y^{* 2}}\right), \\
\mu^{*}(c)=e^{-R c},
\end{gathered}
$$

where $(\cdot)^{*}$ denotes a dimensionless quantity. For notational simplicity, we drop the asterisks from all subsequent quantities. The key dimensionless parameters are the logviscosity ratio and the Peclet number, defined as

$$
R=-\ln \left(\frac{\mu_{1}}{\mu_{2}}\right), \quad \mathrm{Pe}=\frac{U a}{\phi D} .
$$

When the injected fluid is more viscous than the ambient $(R<0)$, the interface is stable and the concentration evolves by diffusion alone, with a classical error-function profile. However, when the injected fluid is less viscous than the ambient $(R>0)$, the interface can be unstable, leading to complex fingering patterns. We focus on the latter problem here. The Peclet number provides a ratio of the characteristic timescales for diffusion and advection: when $\mathrm{Pe} \ll 1$, diffusion dominates the dynamics, and when $\mathrm{Pe} \gg 1$, advection dominates. In the diffusive limit, as will be shown later, the instability can be suppressed so we will, therefore, focus predominantly on the limit $\mathrm{Pe} \gg 1$.

We work in a reference frame moving with the velocity of the injected fluid, and introduce transformed variables

$$
\tilde{u}=u-1, \quad \tilde{x}=x-t .
$$

In this frame, equations $(2.5-2.7)$ become

$$
\begin{gathered}
\frac{\partial \tilde{u}}{\partial \tilde{x}}+\frac{\partial v}{\partial y}=0 \\
-(\tilde{u}+1) \mu=\frac{\partial p}{\partial \tilde{x}}, \quad-v \mu=\frac{\partial p}{\partial y}, \\
\frac{\partial c}{\partial t}+\tilde{u} \frac{\partial c}{\partial \tilde{x}}+v \frac{\partial c}{\partial y}=\frac{1}{\mathrm{Pe}}\left(\frac{\partial^{2} c}{\partial \tilde{x}^{2}}+\frac{\partial^{2} c}{\partial y}\right) .
\end{gathered}
$$

Again, for notational convenience, we drop the tildes from all subsequent quantities.

\subsection{Boundary conditions}

Similar to previous work (Tan \& Homsy 1988), we impose periodicity at the top and bottom boundaries. The upstream and downstream concentration are fixed at $c=1$ and 
$c=0$ respectively and the horizontal velocity is fixed at $u=0$ (in the moving frame). The boundary conditions are thus

$$
\begin{gathered}
c(x, 0, t)=c(x, 1, t), \quad u(x, 0, t)=u(x, 1, t), \quad v(x, 0, t)=v(x, 1, t), \\
c(-\infty, y, t)=1, \quad c(\infty, y, t)=0, \\
u(-\infty, y, t)=u(\infty, y, t)=0 .
\end{gathered}
$$

\subsection{Diagnostic quantities}

As the instability develops and an array of fine fingers form, the local fingering dynamics become chaotic and are controlled by non-linear interactions between fingers. Instead of examining the behaviour of each individual finger, we aim to examine how the fingering dynamics evolve globally. To do so, we compute the average concentration over the transverse direction,

$$
\bar{c}(x, t)=\int_{0}^{1} c(x, y, t) d y .
$$

Using this definition, and defining the deviations $c^{\prime}(x, y)=c(x, y)-\bar{c}(x),(2.13)$ can be written as two coupled equations for the mean and perturbed concentrations,

$$
\begin{gathered}
\frac{\partial \bar{c}}{\partial t}+\frac{\partial \overline{u c^{\prime}}}{\partial x}=\frac{1}{\operatorname{Pe}} \frac{\partial^{2} \bar{c}}{\partial x^{2}} \\
\frac{\partial c^{\prime}}{\partial t}+\frac{\partial u c^{\prime}}{\partial x}+\frac{\partial u \bar{c}}{\partial x}-\frac{\partial \overline{u c^{\prime}}}{\partial x}+\frac{\partial v c^{\prime}}{\partial y}=\frac{1}{\operatorname{Pe}}\left(\frac{\partial^{2} c^{\prime}}{\partial y^{2}}+\frac{\partial^{2} c^{\prime}}{\partial x^{2}}\right) .
\end{gathered}
$$

We will use this decomposition in our derivation of the late-time solution in $\S 6$.

We also examine three global quantities over time: the mixing length $h$, which quantifies the width of the mixing zone; the average number of fingers $n$, which gives an inverse measure of the transverse length scale; and the Nusselt number, $\mathrm{Nu}$, which quantifies the total convective mixing rate. These quantities are defined as,

$$
\begin{gathered}
h=\left.x\right|_{\bar{c}=0.01}-\left.x\right|_{\bar{c}=0.99,} \\
n=\frac{1}{h} \int_{\left.x\right|_{\bar{c}=0.99}}^{\left.x\right|_{\bar{c}=0.01}} \eta(x) d x, \\
\mathrm{Nu}=\int_{-\infty}^{\infty} \int_{0}^{1} u\left(c-\frac{1}{2}\right) d y d x,
\end{gathered}
$$

where the number of fingers $\eta(x)$ is calculated by counting the number of local maxima in a vertical slice. Note that, the Nusselt number is often defined as $\mathrm{Nu}^{*}=1+\mathrm{PeNu}$, which is the ratio between total transport and diffusive transport (Zhou 2013). Here we instead use the Nusselt number simply to quantify the convective transport.

\section{Numerical Method}

A variety of techniques have been used to solve the coupled equations $(2.8,2.11$ 2.13) including spectral (Tan \& Homsy 1988; Zimmerman \& Homsy 1991, 1992; De Wit \& Homsy 1997b), pseudo-spectral (Islam \& Azaiez 2005) and finite-difference methods (Jha et al. 2011a,b). Here we use a modified finite-difference method, which is numerically stable for all $R$. 
Given that the fluids are incompressible, we write the velocity in terms of a streamfunction $\Psi(x, y, t)$,

$$
u=\frac{\partial \Psi}{\partial y}, \quad v=-\frac{\partial \Psi}{\partial x} .
$$

Eliminating the pressure from (2.12) and combining with (3.1) and (2.8) results in a non-linear elliptic equation for the streamfunction,

$$
\frac{\partial^{2} \Psi}{\partial x^{2}}+\frac{\partial^{2} \Psi}{\partial y^{2}}-R \frac{\partial c}{\partial x} \frac{\partial \Psi}{\partial x}-R \frac{\partial c}{\partial y} \frac{\partial \Psi}{\partial y}=R \frac{\partial c}{\partial y},
$$

with boundary conditions

$$
\begin{gathered}
\Psi(x, 0, t)=\Psi(x, 1, t), \\
\Psi(-\infty, y, t)=\Psi(\infty, y, t)=0,
\end{gathered}
$$

from (2.14) and (2.16).

In order to simulate an infinite strip, we impose boundary conditions (2.15) and (3.4) at $x= \pm \Gamma / 2$, where $\Gamma$ is chosen to be sufficiently large such that these boundaries are far from the fingered region. Furthermore, since previous work has shown that solutions are independent of the aspect ratio as long as the fingered region is sufficiently far from the boundaries (Ruith \& Meiburg 2000; Tan \& Homsy 1988), we use a growing domain to minimize computational time. Each simulation is initialized with a domain length $\Gamma=1$, and $\Gamma$ is doubled whenever $\bar{c}(x=-0.3 \Gamma)=0.999$ or $\bar{c}(x=0.3 \Gamma)=0.001$. We compared simulations with variable and fixed domain sizes to confirm that this mapping had no measurable effect on the dynamics.

We discretise the domain on a rectangular grid with $\left(n_{x}, n_{y}\right)$ grid points in the $(x, y)$ direction. Each simulation is initialized with an almost sharp interface and an added small random perturbation centred at $x=0$,

$$
c_{0}=\frac{1}{2}+\frac{1}{2} \operatorname{erf}\left(-\frac{x}{\sqrt{t_{0}}}\right)+r(x, y) e^{-x^{2} / t_{0}},
$$

where the function $r(x, y)$ returns a uniformly distributed random number on the interval $\left[0,10^{-5}\right]$. The diffusive error function with small effective time origin $t_{0}$ is included in (3.5) to aid the accuracy of the numerical scheme at early times. We set $t_{0}=10^{-6}$ in all simulations.

At each time step, we solve (3.2) using an iterative multi-grid solver (Adams 1999) with the solution at the previous time step used as an initial guess. We use sixth-order compact finite differences (Lele 1992) to discretise the spatial derivatives in (3.2) and (2.13), and advance (2.13) in time using a third-order Runge-Kutta scheme. We select the time step, $\delta t=\min \left(10^{-3}, \min \left(\frac{\delta x}{u_{\max }}, \frac{\delta y}{v_{\max }}\right)\right)$, to always satisfy the Courant-FriedrichsLewy condition. To validate the numerical method, we tested the convergence of the solution with increased spatial and temporal resolution and compared the growth rate of single-mode perturbations to the linear stability theory of Tan \& Homsy (1986).

\section{Fingering pattern and regimes}

Figure 2 shows a sequence of snapshots from a typical simulation for log-viscosity ratio $R=2$ and Peclet number $\mathrm{Pe}=2000$. At early times, the initially very sharp interface begins to smooth out and a series of fine fingers develop (figure 2a). At intermediate times, once the fingers reach a certain size, they begin to interact, which drives coarsening in the vertical direction and growth in the horizontal direction (figures 2b,c,d). Overall, these 
a)

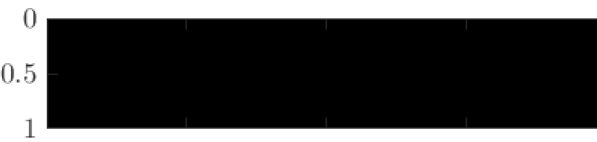

b) 0

0

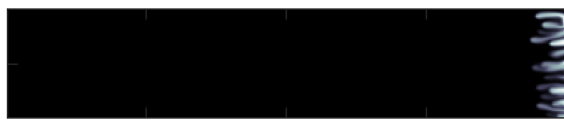

c) 0.5
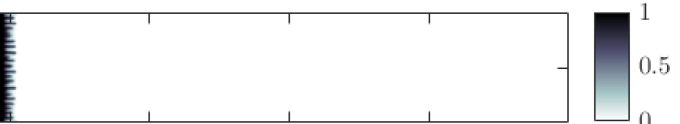

.

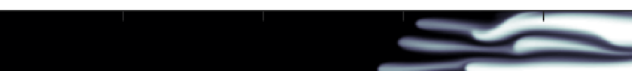

2

d) 0 .

${ }^{0}$

cras

1

e) 0.5

0.5

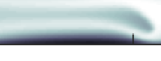

$-4$
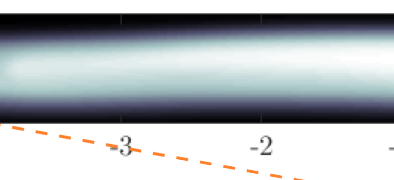

$-1$

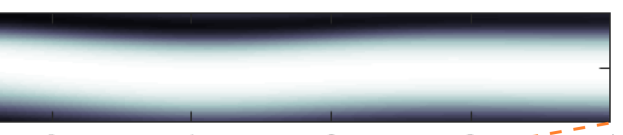

0
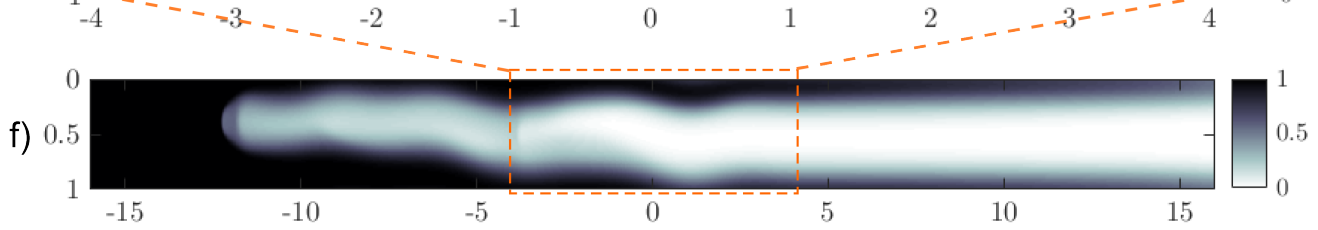

Figure 2. Colourmaps of the concentration field (in a frame moving with the interface) over the course of a simulation. Here, $R=2$ and $\mathrm{Pe}=2000$. Snapshots, from top to bottom, are taken at (a) $t=0.5$, (b) $t=1$, (c) $t=3$, (d) $t=10$, (e,f) $t=31$. Note that the numerical domain is significantly larger than shown in the lower 3 panels. Panel (f) is zoomed out to include the full finger and note that the figure is horizontally compressed by a factor of 4 .

non-linear interactions lead to coalescence until a single broad finger remains (figures $2 \mathrm{e}, \mathrm{f})$.

All of our numerical simulations, which have Pe ranging from 100 to 16000 and $R$ ranging from 1 to 5 , show this qualitative behaviour. In general, we find that at early times the interface diffuses and a set of fingers develop. The number of fingers that develop increases with both the Peclet number and the log-viscosity ratio. The fingers then reach a critical size after which they interact non-linearly. The fingers interact via a variety of different mechanisms. These include shielding, when a longer finger widens at the tip and shields the growth of smaller neighbouring ones; fading, when a finger stops growing and diffuses into the ambient; and coalescence, when two or more fingers merge together. When the Peclet number and log-viscosity ratio are large, the fingers also exhibit more complex behaviour including tip-splitting, when a finger splits into two at the tip; and branching, when a finger sheds fingers from its side (Islam \& Azaiez 2005; Tan \& Homsy 1988; Zimmerman \& Homsy 1991).

Regardless of the Peclet number and log-viscosity ratio, these interactions, on aggregate, lead to coalescence until a single broad finger remains. This finding is contrary to previous suggestions that the final state may include multiple fingers. The single finger that remains diffuses while propagating at an exponentially slowing speed, ultimately leaving a linear background concentration gradient that is gradually smoothed out by diffusion. We find that the final mixing zone length increases with both $R$ and Pe.

Figure 3 shows the mixing length $h$, number of fingers $n$, and Nusselt number $\mathrm{Nu}$ as functions of time for different Peclet numbers (left) and log-viscosity ratios (right). 

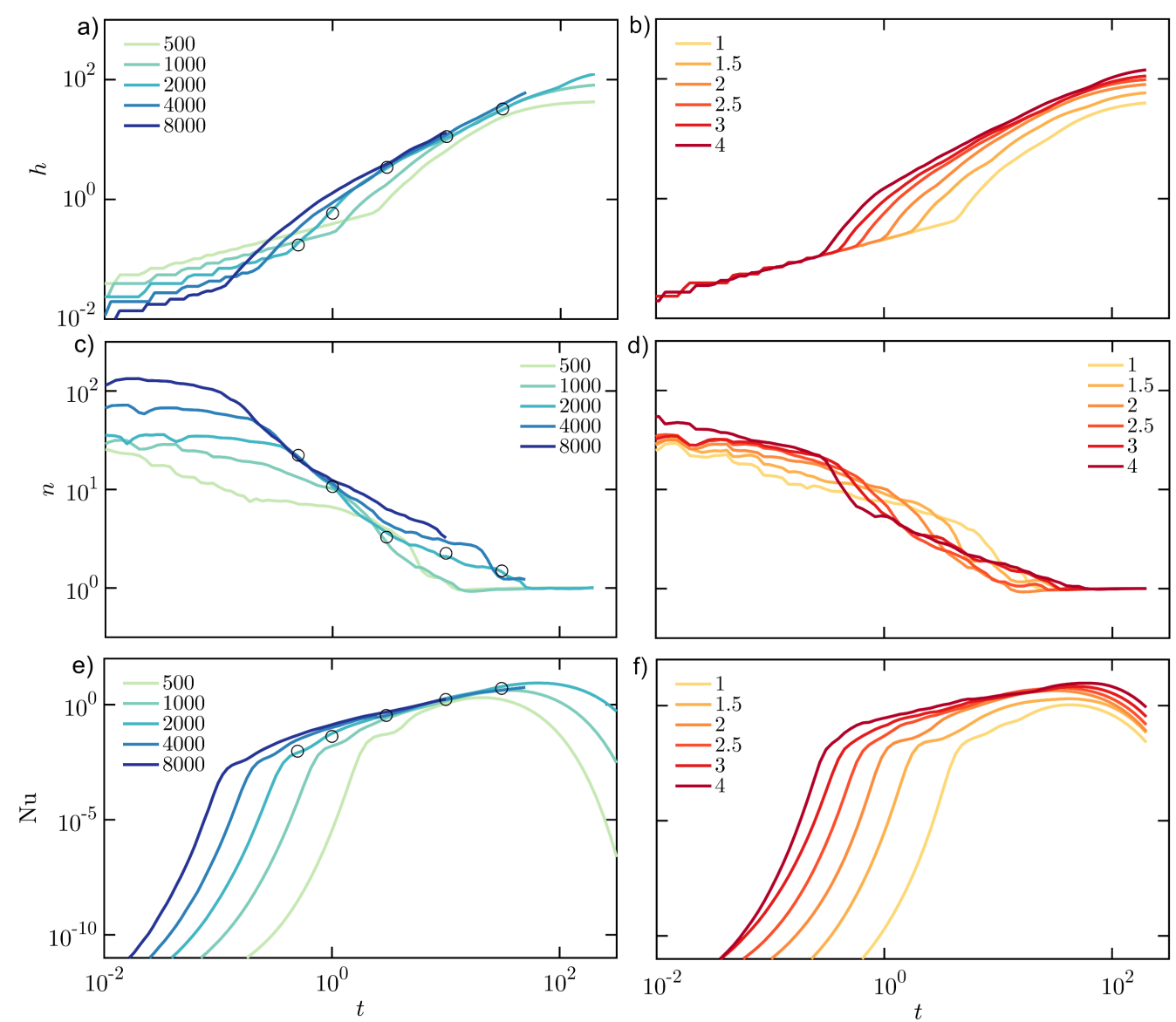

Figure 3. Plots of $(\mathrm{a}, \mathrm{b})$ the mixing length $h,(\mathrm{c}, \mathrm{d})$ the number of fingers $n$, and $(\mathrm{e}, \mathrm{f})$ the Nusselt number $\mathrm{Nu}$ as functions of time plotted on logarithmic axes. To reduce the noise in the data, two different simulations are averaged. (a,c,e) Data for log-viscosity ratio $R=2$ and different Peclet numbers Pe as marked. The black circles correspond to the snapshots in figure 2. (b,d,f) Data for $\mathrm{Pe}=1000$ and different values of $R$ as marked.

Figures 3a,b show that the mixing length initially grows, then steepens, before finally slowing towards a constant. The early-time mixing length is larger for small Peclet numbers and is independent of the log-viscosity ratio whereas the final mixing length increases with both the Peclet number and log-viscosity ratio. Figures 3c,d show the average number of fingers is fairly constant at early times, decays to one at intermediate times, and stays constant at one at late times. Although the initial number of fingers increases with the Peclet number and log-viscosity ratio, the flow always tends to a single finger eventually, irrespective of the parameters. Finally, figures $3 \mathrm{e}$,f show that the Nusselt number first grows exponentially, then grows more slowly and finally decays exponentially.

Based on these sets of observations we partition the flow into three distinct regimes: (i) an early-time, linearly unstable regime: the mixing zone grows diffusively and fingers grow exponentially; (ii) an intermediate-time non-linear regime: fingers coalesce and the mixing length and Nusselt number exhibit power-law growth; and, (iii) a late-time, single-finger, exchange-flow regime: a single pair of counter-propagating fingers slow exponentially. 

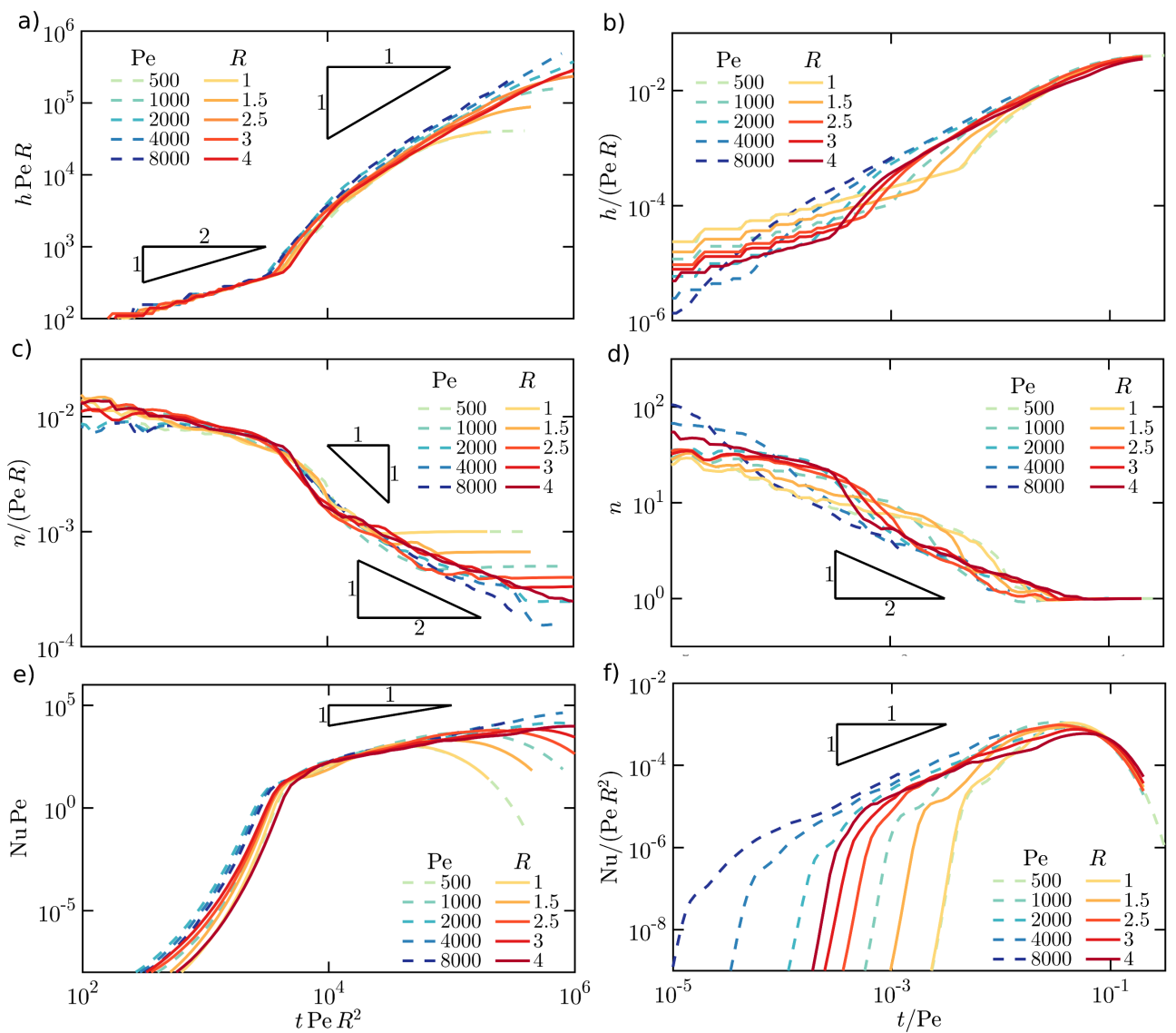

Figure 4. Rescaled plots of (a,b) $h,(\mathrm{c}, \mathrm{d}) n$, and (e,f) Nu for early times (left) and late times (right). The dashed lines are for constant $R=2$ and different Pe as marked, while the solid lines are for constant $\mathrm{Pe}=1000$ and different $R$ as marked. To reduce the noise in the data, two different simulations are averaged.

Each regime shows different dynamics and exhibits different scalings. We explore these scalings in the following subsection, before examining each regime in more detail in sections 5 and 6 .

\subsection{Scalings}

At the start of all simulations, the interface is relatively sharp and the concentration and velocity perturbations are small. Diffusion across the interface dominates the growth of the mixing zone, and a diffusive balance $\frac{c}{t} \sim \frac{c}{\mathrm{Pe} h^{2}}$ gives the scaling for the mixing length $h \sim\left(\frac{t}{\mathrm{Pe}}\right)^{\frac{1}{2}}$, as can be seen in figure 4a. In this linearly unstable regime, the aspect ratio of the fingers is $O(1)$; hence, from incompressibility, $\frac{u}{x} \sim \frac{v}{y} \Rightarrow u \sim v$. The linearized elliptic equation (3.2) further suggests a balance $\frac{u}{y} \sim \frac{v}{x} \sim \frac{R c}{y}$, or $u \sim v \sim R$. The linear scaling of the velocity with the log-viscosity ratio, together with an advection-diffusion balance in (2.13), indicates that $\frac{c}{t} \sim \frac{u c}{x} \sim \frac{c}{\mathrm{Pe} x^{2}}$, or $t \sim \frac{1}{R^{2} \mathrm{Pe}}$ and $x \sim \frac{1}{R \mathrm{Pe}}$. That is, at early times, the number of fingers scales linearly with both $R$ and Pe. Figure $4 \mathrm{c}$ shows a rescaled plot of the number of fingers which collapses well with this scaling. Finally, the Nusselt number is defined as the product of the exponentially growing velocity $u \sim R e^{\sigma t}$ and concentration perturbations $c^{\prime} \sim e^{\sigma t}$ integrated over the size of the perturbations $x \sim \frac{1}{R \mathrm{Pe}}$ (where $\sigma$ is 


\begin{tabular}{c|c|c} 
Early times & Intermediate times & Late times \\
\hline$h \sim\left(\frac{t}{\mathrm{Pe}}\right)^{\frac{1}{2}}$ & $h \sim R t$ & $h \sim R \mathrm{Pe}$ \\
$n \sim R \mathrm{Pe}$ & $n \sim \frac{1}{R t} \quad n \sim\left(\frac{t}{\mathrm{Pe}}\right)^{\frac{1}{2}}$ & $n=1$ \\
$\mathrm{Nu} \sim e^{t R^{2} \mathrm{Pe}} / \mathrm{Pe}$ & $\mathrm{Nu} \sim R^{2} t$ & $\mathrm{Nu} \sim R^{2} \mathrm{Pe} e^{-\mathrm{Pe} t}$
\end{tabular}

TABLE 1. Scalings for $h, n$, and Nu for early, intermediate, and late times. The transition from the early-time to intermediate-time regime occurs at $t \sim O\left(\frac{1}{R^{2} \mathrm{Pe}}\right)$ and the transition from the intermediate-time to late-time regime occurs at $t \sim O(\mathrm{Pe})$.

the growth rate of the instability). Given the time scale identified above, $t \sim \frac{1}{R^{2} \mathrm{Pe}}$, we collapse the data for the Nusselt number with the scaling $\mathrm{Nu} \sim e^{t R^{2} \mathrm{Pe}} / \mathrm{Pe}$ (figure $4 \mathrm{e}$ ).

At intermediate times the fingers interact non-linearly causing them to elongate and coarsen. The horizontal velocity remains relatively constant and is solely a function of the log-viscosity ratio, $u=U(R)$. An advective balance in equation (2.13) gives the scaling $\frac{U c}{h} \sim \frac{c}{t}$, or $h \sim U(R) t$, and this linear growth of the mixing zone in time can be seen in figures 4a,b. In fact, we return to the functional form of the velocity $U(R)$ in section 5.2 , and find that it can be approximated by $U \sim R$ for small $R$. The number of fingers $n$, in the intermediate-regime, follows two distinct coalescence regimes. Initially the coalescence is advectively dominated, and in this limit (2.13) gives the scaling $\frac{v c}{1 / n} \sim \frac{c}{t}$. Assuming that the transverse velocity is $O(R)$ and constant, then $n \sim \frac{1}{R t}$. Subsequently, the flow becomes diffusively dominated and (2.13) gives the scaling $\frac{c}{t} \sim \frac{c}{\mathrm{Pe} / n^{2}} \Rightarrow n \sim(t / \mathrm{Pe})^{-\frac{1}{2}}$. These two scaling laws can be seen in figures $4 \mathrm{c}, \mathrm{d}$. In the intermediate-time regime, the Nusselt number scales with the width of the mixing region $(h \sim R t)$ and the average convective flux, which scales with the velocity $U \sim R$. Together, this gives the scaling $\mathrm{Nu} \sim R^{2} t$ (see figures $4 \mathrm{e}, \mathrm{f}$ ). These observations suggest that the Nusselt number and growth of the mixing zone are independent of the Peclet number and, after a small amount of time spent advectively coalescing, the finger coalescence becomes independent of the viscosity ratio.

Finally, at late times, a single pair of long, thin fingers counter-propagate and decay through a background concentration gradient. As seen in figure $4 \mathrm{~d}$, all simulations tend to this single-pair (single-maxima) state. Assuming that the concentration deviations from the background are small and applying a long, thin approximation to equation (3.2), results in the scaling $u \sim R$ (as discussed in more detail in $\S 6$ ). Balancing longitudinal advection and transverse diffusion over a single finger yields the scaling $\frac{R c}{h} \sim \frac{1}{\mathrm{Pe}} \Rightarrow$ $h \sim R$ Pe. This tendency towards a constant mixing length proportional to $R P$ e is shown in figure $4 \mathrm{~b}$. A diffusive balance, $\frac{c}{t} \sim \frac{1}{\mathrm{Pe}}$, suggests that the time should be scaled by the Peclet number in this late-time regime. Applying the same argument as before, the Nusselt number decays exponentially like $\mathrm{Nu} \sim \mathrm{Pe} R^{2} e^{-\mathrm{Pe} t}$.

The scalings are summarized in table 1 . In the following sections, we discuss each of these regimes in more detail with an emphasis placed on understanding the evolution of the transversely averaged concentration. 

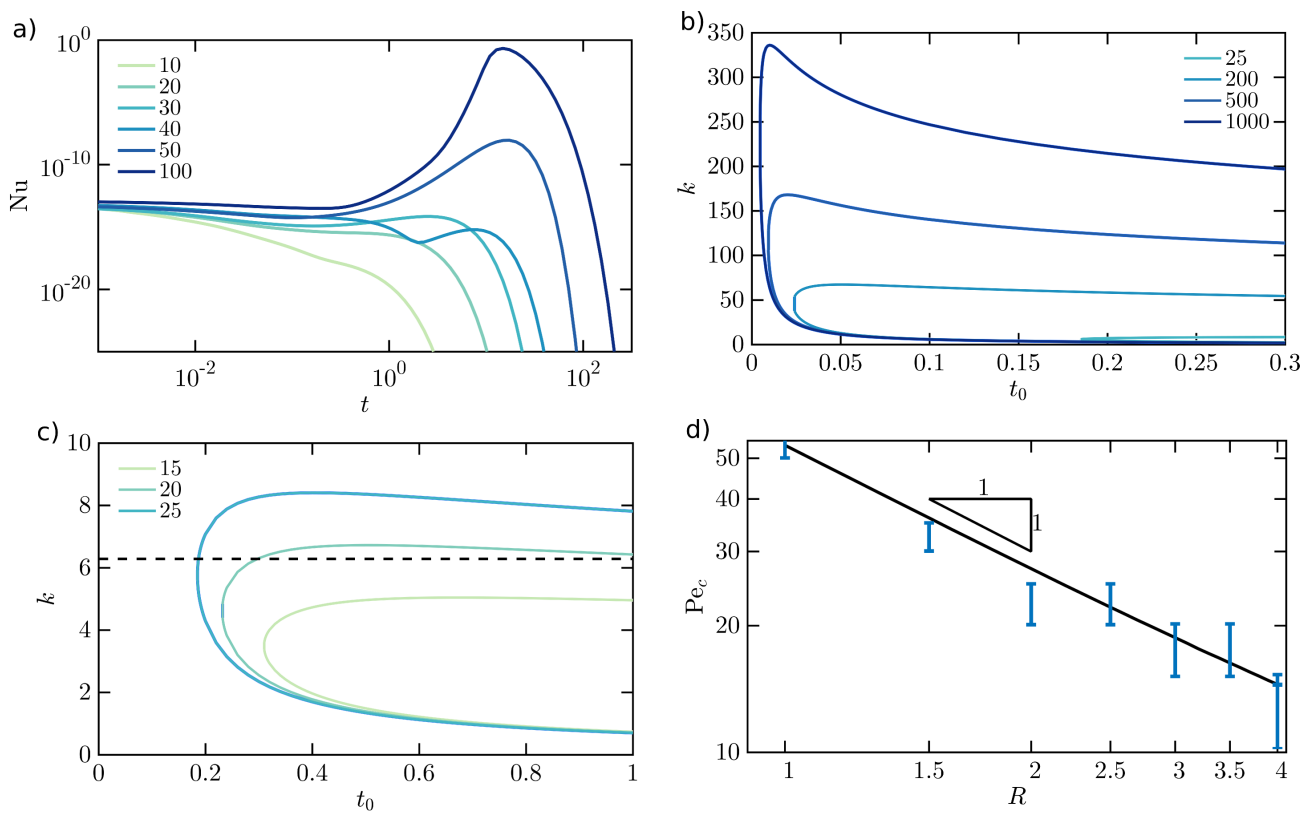

Figure 5. (a) $\mathrm{Nu}(t)$, attained from direct numerical simulations, plotted on logarithmic axes for $R=2$ and different $\mathrm{Pe}$ as marked. The Nusselt number is strictly decreasing for $\mathrm{Pe}=20$, but has a period of growth for $\mathrm{Pe}=30$, suggesting a point of marginal stability between $\mathrm{Pe}=20$ and 30. (b,c) Marginal stability curves for $R=3$ and (b) $\mathrm{Pe}=25,200,500,1000$ (c) $\mathrm{Pe}=15,20,25$. (d) Plot of the critical Peclet number versus log-viscosity ratio based on the linear stability analysis (black line) and numerical simulations (blue ranges). The lower and upper estimated values of $\mathrm{Pe}_{c}$ from our simulations in (d) are given, respectively, by the largest $\mathrm{Pe}$ for which $\mathrm{Nu}(t)$ monotonically decreases, and by the smallest Pe for which $\mathrm{Nu}(t)$ increases at any time.

\section{Early- and intermediate-time regimes}

\subsection{Early times: linearly unstable regime}

The concentration gradient between the two fluids, which are not moving relative to each other, is initially very high and spreads by diffusion. Neglecting the very small initial perturbations in (3.5), the resultant concentration profile is one-dimensional and given by

$$
c(x, t)=\frac{1}{2}+\frac{1}{2} \operatorname{erf}\left(-\frac{x}{\sqrt{4 t / \mathrm{Pe}}}\right) .
$$

Therefore, before the instability manifests itself, the concentration front widens like $(t / \mathrm{Pe})^{\frac{1}{2}}$, which corresponds to the early-time scaling of $h$ (see figure $4 \mathrm{a}$ ).

When $R>0$, the flow rapidly develops a viscous-fingering instability in which perturbations grow exponentially. Many authors have explored the onset of viscous fingering in a variety of contexts using linear stability theory. Tan \& Homsy (1987) found that the instability can be suppressed for all times, in a radial geometry, if the Peclet number is below some critical value. In a planar geometry, however, Pramanik \& Mishra (2015a) found a time-dependent critical Peclet number which decreases in time, and suggested that there may be no Peclet number for which the flow is always stable.

In this section, we show that there is, in fact, a critical Peclet number below which the flow is always stable in a planar geometry. To motivate the existence of this critical 
Peclet number, figure 5 a shows $\mathrm{Nu}(t)$ for $R=2$ and small Peclet numbers. For the range of Peclet numbers plotted, the Nusselt number never transitions to power-law growth, suggesting that there are choices of parameters where the flow never enters the non-linear regime. In fact, we notice that for Peclet numbers less than or equal to 20, the Nusselt number is strictly decreasing, implying the configuration is stable for all times, while for Peclet numbers greater than or equal to 30, the Nusselt number goes through a period of growth. In this section, we perform a linear stability analysis to show the existence of a critical Peclet number for the instability.

We start with a diffusive base-state solution of the unperturbed system $c_{0}(x, t)$ given by equation (5.1). To accommodate the rapidly varying base-state at early times we use a similarity transformation $\xi=x / \sqrt{t}$, in terms of which (5.1) is steady,

$$
c_{0}(\xi)=\frac{1}{2}\left[1+\operatorname{erf}\left(\frac{-\xi \sqrt{\mathrm{Pe}}}{2}\right)\right] .
$$

We then linearize equations (2.13), (3.1), and (3.2) about this base-state and look for perturbations of the form $u^{\prime}(\xi, y, t)=\phi(\xi) \tau(t) e^{i k y}$ and $c^{\prime}(\xi, y, t)=\beta(\xi) \tau(t) e^{i k y}$, which satisfy,

$$
\begin{gathered}
\left(\sigma\left(t_{0}\right)-\frac{\xi}{2 t_{0}} \frac{d}{d \xi}-\frac{1}{\operatorname{Pe} t_{0}} \frac{d^{2}}{d \xi^{2}}+\frac{k^{2}}{\mathrm{Pe}}\right) \beta=-\frac{1}{\sqrt{t_{0}}} \frac{d c_{0}}{d \xi} \phi \\
\left(\frac{1}{t_{0}} \frac{d^{2}}{d \xi^{2}}-\frac{R}{t_{0}} \frac{d c_{0}}{d \xi} \frac{d}{d \xi}-k^{2}\right) \phi=-R k^{2} \beta
\end{gathered}
$$

where $\left.\sigma\left(t_{0}\right) \equiv \frac{1}{\tau} \frac{d \tau}{d t}\right|_{t=t_{0}}$ is the instantaneous growth rate at $t=t_{0}$, such that $\tau=e^{\int_{0}^{t} \sigma d t_{0}}$ (see also Pramanik \& Mishra 2015a). We note that this formulation does not require any assumption of a slowly-varying or quasi-steady background. We solve (5.3) and (5.4) by discretizing the domain using standard second-order finite-difference approximations for the differential operators, which yields the matrix eigenvalue problem

$$
\mathbf{M} \beta=\sigma \beta .
$$

The growth rate of the most unstable mode is given by the maximum eigenvalue of the matrix $\mathbf{M}$. This growth rate depends on Pe and $R$, as well as, time $t_{0}$ and the wavenumber of the perturbation $k$.

Figure $5 \mathrm{~b}$ shows the marginal stability curve $\sigma\left(k, t_{0}\right)=0$, where $\sigma$ is the growth rate of the most unstable mode, for $R=2$ and a variety of Peclet numbers. The system is always initially stable and goes unstable at a critical time $t_{0}^{*}>0$. Zooming into the region around wavenumber $k=2 \pi$ (figure $5 \mathrm{c}$ ), which is the largest mode that is permissible inside the domain, we notice that for $\mathrm{Pe}=20$, the marginal stability curve lies above $k=2 \pi$ for only a finite amount of time: once the marginal stability curve falls below this value, the flow is again stable. In fact, this transition back to stability at large $t_{0}$ is a general feature for all $R$ and $P e$ and this intermittent stability suggests that if the interface is initially diffuse enough, the instability can be suppressed. Finally, we notice that for Peclet numbers smaller than some critical value $\operatorname{Pe}_{c}(R)$, the growth rate is only positive for wavenumbers smaller than $2 \pi$. These modes do not fit in the domain and the interface is therefore always stable. For example, in figure $5 \mathrm{c}$ the critical Peclet number lies between 15 and 20 .

The transitions out of, and back into, stability, occur as diffusion tends to arrest the instability. The system is initially stable because, for small $t$, the growth of the interface $\left(O\left(t^{-\frac{1}{2}}\right)\right)$ outpaces the exponential growth of the perturbations. Matching the diffusive length-scale to the length-scale of the most unstable perturbation gives a transition time 

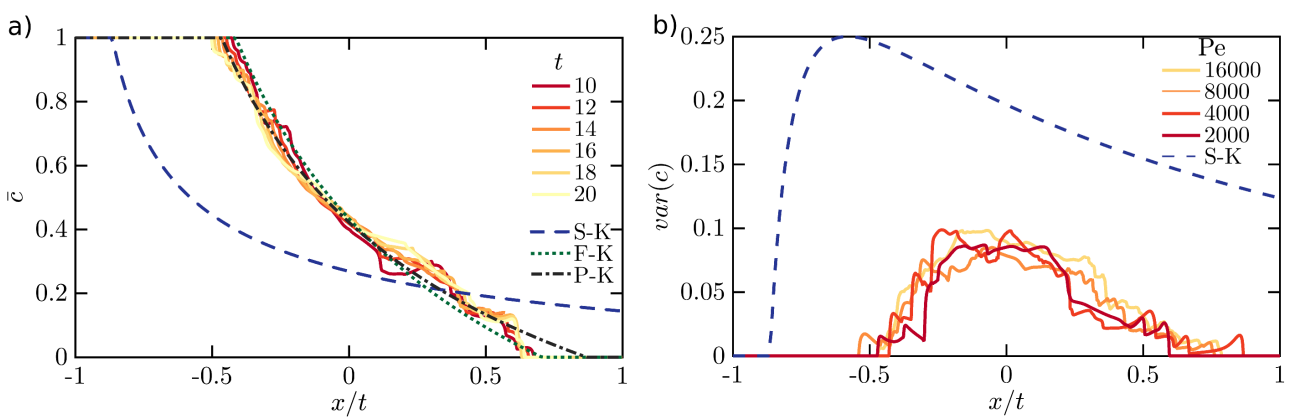

Figure 6. (a) Plot of the transveresely averaged concentration against the similarity variable $x / t$. Here $R=2, \mathrm{Pe}=1000$ and the time, given by the colour, ranges from 10 to 20. Each curve plotted represents the average of five different simulations. The dashed lines represent the three different model solutions: simple Koval (blue, dashed), fitted Koval (green, dotted), and parabolic Koval (black, dot-dashed) . (b) Plot of the transverse variance in concentration for $\mathrm{Pe}=2000,4000,8000$, and 16000 at $t=8,4,2$ and 1 . By sampling at these different times, we normalize for the effect of the onset of the instability. The variance calculated from the simple Koval model is given by the blue dashed line.

$t \sim \frac{1}{R^{2} \mathrm{Pe}}$. At sufficiently large times, the base flow is again stable, because the background concentration gradient has weakened to such an extent that transverse diffusion can smear out the advective growth of perturbations. Balancing these two terms for $x, y \sim O(1)$ gives a transition time back to stability of $t \sim \frac{1}{\mathrm{Pe}}$. At some critical Peclet number, $\mathrm{Pe}_{c}$, these two time-scales become the same, and the instability is completely suppressed. This balance gives $\mathrm{Pe}_{c} \sim \frac{1}{R}$.

Figure 5d shows $\operatorname{Pe}_{c}(R)$ calculated from the linear stability analysis, which agrees with this predicted scaling. The figure also shows estimates of $P e_{c}$ from direct numerical simulations, which give a reasonable agreement with the theory.

\subsection{Intermediate times: non-linear coalescence regime}

The linear instability results in a number of fingers which grow exponentially and independently of their neighbours. After some time, the fingers begin to interact with each other. Although the non-linear finger interactions exhibit complex and chaotic patterns and vary significantly over time and from simulation to simulation, the number of fingers, mixing length, and Nusselt number are largely indifferent to the exact intermediary mechanisms (see rescaled data in figure 4). The transversely averaged concentration is asymmetric, non-linear, and evolves in a self-similar fashion (figure 6a). There have been many attempts to model the behaviour of the transversely averaged concentration, with one of the simplest and most widely used models being the empirically derived formula of Koval (1963). While this model has been revisited by multiple authors (Booth 2010; Yortsos \& Salin 2006), a fully closed model is yet to be derived. In this section we start by re-deriving the simple model that was first proposed by Koval (hereafter, the 'simple Koval model'), and comment on its strengths and shortcomings. In order to address one of these shortcomings, we then propose a simple improvement to the model, which gives a qualitative improvement when compared with the numerical simulations.

The simple Koval model can be derived in the limit where both the aspect ratio of the fingers and the Peclet number are large, $(h n \gg 1$ and $\mathrm{Pe} \gg 1$ respectively). Under these conditions the flow is predominantly horizontal and longitudinal diffusion is negligible. 
The velocity is calculated by taking the leading order expansion in $h n$ in (3.1),

$$
\frac{\partial u}{\partial y}-R u \frac{\partial c}{\partial y}=R \frac{\partial c}{\partial y}
$$

which has solution

$$
u=\frac{e^{R c}}{\int_{0}^{1} e^{R c} d y}-1 .
$$

Substituting this form for the velocity into (2.18) and neglecting longitudinal diffusion gives,

$$
\frac{\partial \bar{c}}{\partial t}+\frac{\partial}{\partial x}\left(\frac{\int_{0}^{1} c e^{R c} d y}{\int_{0}^{1} e^{R c} d y}-\bar{c}\right)=0 .
$$

The simple Koval model proceeds under the assumption that the fingered region consists of $\eta_{b}(x)$ leftward-propagating fingers of width $w_{b}(x)$ with uniform concentration $\mathrm{c}=0$ and $\eta_{f}(x)$ rightward-propagating fingers of width $w_{f}(x)$ with uniform concentration $\mathrm{c}=1$. Under these assumptions, (5.8) becomes

$$
\frac{\partial \bar{c}}{\partial t}+\frac{\partial}{\partial x}\left(\frac{\eta_{f} \int_{-w_{f}}^{w_{f}} e^{R} d y}{\eta_{f} \int_{-w_{f}}^{w_{f}} e^{R} d y+\eta_{b} \int_{-w_{b}}^{w_{b}} 1 d y}-\bar{c}\right)=0 .
$$

In addition, the total area of the fingers has to add up to one, $\eta_{f} w_{f}+\eta_{b} w_{b}=1$, and the total concentration in the forward propagating fingers has to equal the transverse average, $\eta_{f} w_{f}=\bar{c}$. Combining these constraints and simplifying (5.9) results in a hyperbolic equation for $\bar{c}$,

$$
\frac{\partial \bar{c}}{\partial t}+\frac{\partial}{\partial x}\left(\frac{M \bar{c}}{M \bar{c}+1-\bar{c}}-\bar{c}\right)=0,
$$

where $M \equiv e^{R}=\mu_{2} / \mu_{1}$ is the viscosity ratio between the two unmixed fluids. The solution to $(5.10)$ is

$$
\bar{c}(x, t)= \begin{cases}1 & x / t<\frac{1}{M}-1 \\ \frac{1}{M-1}\left(\sqrt{\frac{M}{x / t+1}}-1\right) & \frac{1}{M}-1 \leqslant x / t \leqslant M-1 \\ 0 & x / t>M-1 .\end{cases}
$$

Figure 6a compares this solution to the numerical simulations. The simple Koval model accurately predicts two qualitative features of the non-linear spreading process: an asymmetric concentration profile, and self-similarity in the variable $x / t$. However, this model greatly over-predicts the spreading of the mixing zone (figure 6a). To account for the difference between the model and experiments, Koval, in his original work, empirically fit an effective viscosity $M_{e}$ to the experiments of Blackwell et al. (1959), yielding,

$$
M_{e}=\left[0.22 e^{R / 4}+(1-0.22)\right]^{4} \text {. }
$$

The prediction of (5.11) with $M$ replaced by $M_{e}$ in (5.12), which we denote the 'fitted Koval' model, gives a remarkably good fit with our numerical results (figure 6a). Indeed, the agreement in figure $6 \mathrm{a}$ is all the more surprising given that (5.12) was fitted for fluids with a different relationship between viscosity and concentration than we are using here. Nonetheless, in spite of recent attempts, there is no rigorous derivation of this form of effective viscosity ratio. Furthermore, this fitted model tends to break down for large $M$ (Malhotra et al. 2015).

One of the critical assumptions of the Koval model is that the concentration is either 

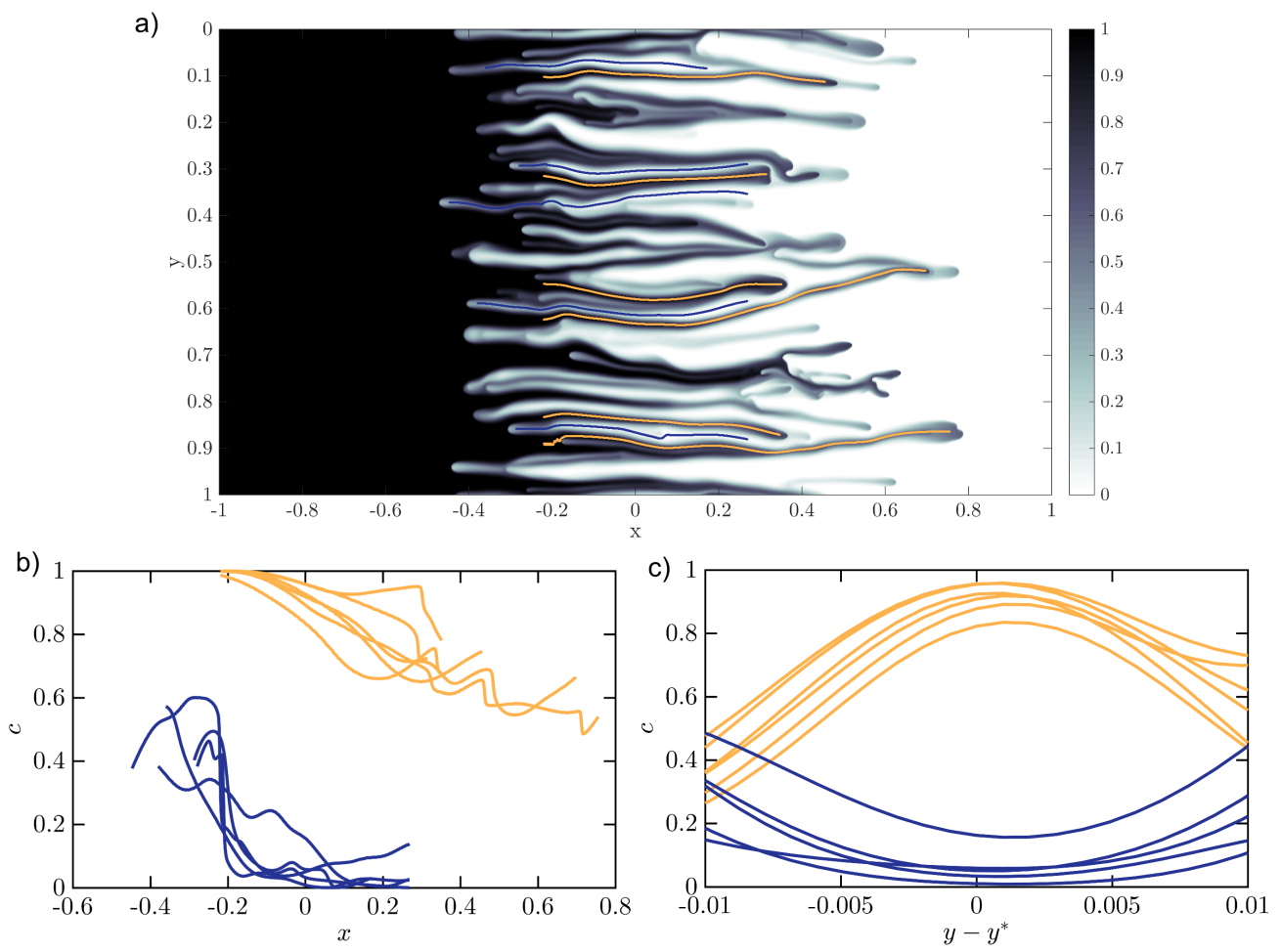

Figure 7. a) Snapshot of the concentration profile at $t=1$ for a simulation with $\mathrm{Pe}=16000$ and $R=2$. Superimposed are lines which follow the peaks (orange) and troughs (blue) in concentration. b) Concentration profile along peaks (orange) and troughs (blue). c) Concentration profile in the transverse direction at $x=0$ centred around the peaks (orange) and troughs (blue).

exactly one or exactly zero. We interrogate this assumption by plotting the concentration field from a simulation with a large Peclet number $(\mathrm{Pe}=16000)$ in figure $7 \mathrm{a}$. We find that even at very large Pe, the concentration is not just one or zero but varies in both the horizontal and vertical direction. The concentration along the local maxima and minima of the fingers (figure $7 \mathrm{~b}$ ) decreases and increases towards the tips, respectively. In the transverse direction, the concentration has roughly parabolic rather than step profiles (figure 7c). These two factors together result in a much smaller prediction for the transverse variance in concentration than the simple Koval model predicts (figure 6b). Interestingly, in this limit of large Pe, the variance is independent of the Peclet number, which suggests that the Peclet number has no effect on the effective viscosity in this regime in agreement with the fact that (5.11) and (5.12) have no dependence on Pe.

Motivated by these observations, we suggest a very simple improvement to the simple Koval model, which addresses one its main assumptions. In the simple Koval model, the viscosity is uniformly given by $e^{R}$ or 1 in each finger, which follows from the assumption of uniform concentration in each finger. However, we observe that the concentration actually varies in a roughly parabolic manner across the fingers. In fact, the significant improvement to the simple model by the empirical fit (5.12) suggests that the main consequence of ignoring this variation is an inaccurate calculation of the effective viscosity. We therefore propose a simple modification of the Koval model in which the viscosity varies with a quadratic concentration profile across each finger; that 


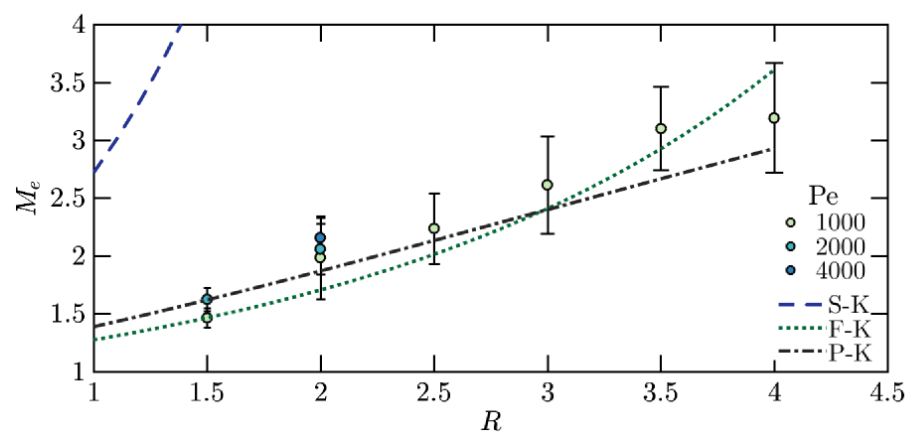

Figure 8. Plot of the effective viscosity ratio measured for different values of $R$ and Pe. Each point plotted is calculated by extracting the value of $M$ from a least-squares fit of (5.11) to the transversely averaged concentration profiles $\bar{c}(x, t)$, at five different times $t=10,11,12,13,14$ and five different simulations. These measurements are then averaged and the error bars represent one standard deviation in these measurements. The three different model predictions are: simple Koval (blue, dashed), fitted Koval (green, dotted), and parabolic Koval (black, dot-dashed)

is, $\mu(y)=e^{R\left(1-y^{2} / w_{f}^{2}\right)}$ and $\mu(y)=e^{R\left(y^{2} / w_{b}^{2}\right)}$ in the forward and backward propagating fingers, respectively. In all other respects, we retain the same assumptions as in the simple Koval model: the fingers are still assumed to be horizontally uniform, and to obtain a simple analytical solution with the same functional form as the simple Koval model, the mean concentration in each finger is still assumed to be either zero or one.

Under these assumptions, (5.9) instead becomes

$$
\frac{\partial \bar{c}}{\partial t}+\frac{\partial}{\partial x}\left(\frac{\eta_{f} \int_{-w_{f}}^{w_{f}} e^{R\left(1-y^{2} / w_{f}^{2}\right)} d y}{\eta_{f} \int_{-w_{f}}^{w_{f}} e^{R\left(1-y^{2} / w_{f}^{2}\right)} d y+\eta_{b} \int_{-w_{b}}^{w_{b}} e^{R\left(y^{2} / w_{b}^{2}\right)} d y}-\bar{c}\right)=0 .
$$

Combining (5.13) with the same constraints as before, and solving, results in the same expression for $\bar{c}$ as (5.11) but with an effective viscosity ratio

$$
M_{e}=\frac{e^{R} \operatorname{erf}(\sqrt{R})}{\operatorname{erfi}(\sqrt{R})},
$$

where erfi $(x)$ is the imaginary error function. We denote this model as the "parabolic Koval' model. As with the original Koval model, the effective viscosity does not depend on the width or the number of fingers.

Figure 8 plots the effective viscosity ratio extracted from the numerical simulations (dots), together with the predictions of the simple Koval model (S-K), the empirically fit effective viscosity (F-K) (5.12), and the analytically derived model with parabolic transverse profiles (P-K) (5.14). The simple Koval model overpredicts the effective viscosity of the fingered region, whereas the parabolic model agrees well with both the empirical fit and numerical experiments. In fact, the parabolic model predicts smaller effective viscosities than the empirical fit for $R>4$ in qualitative agreement with experiments by Malhotra et al. (2015). Although the model agrees well with the data, it remains, of course, an approximation: it does not take into account the along-flow variations in concentration, and it still assumes the concentration (but not the viscosity) is either one or zero in each finger. Nevertheless, we have shown that an accurate effective viscosity in the Koval model can be derived simply by assuming the viscosity varies 


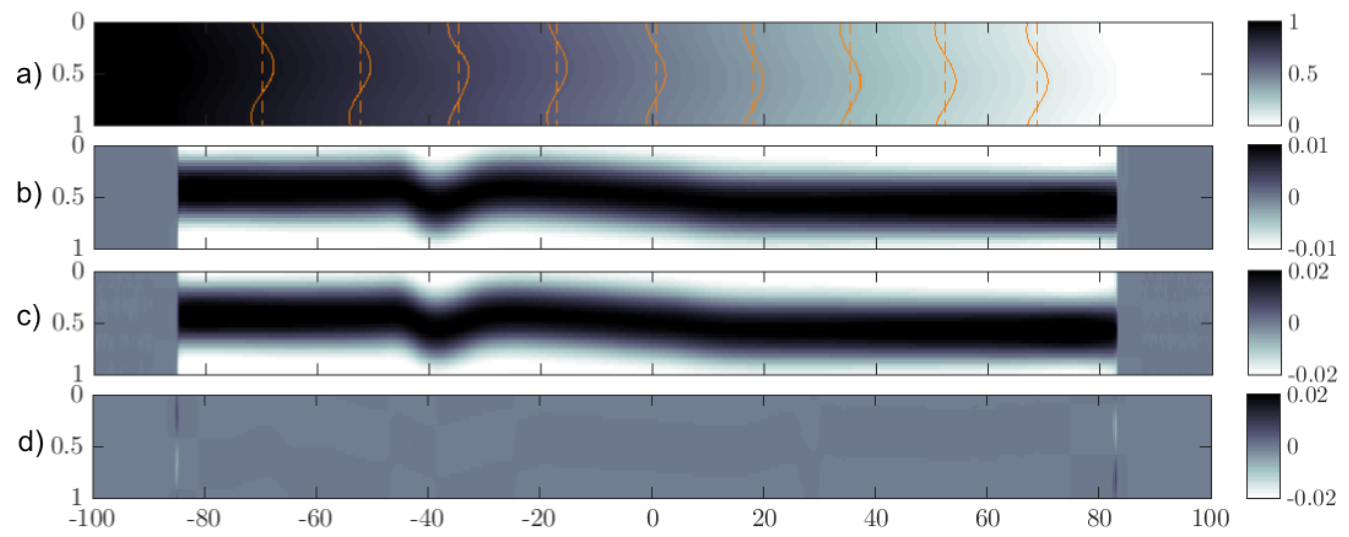

Figure 9. Snapshots at $t=500$ for $R=2, \mathrm{Pe}=2000$. (a) Colourmap of the concentration with overlain contours of the raw (solid) and transversely averaged (dashed) concentration. (b) Colourmap of $c^{\prime}(x, y)=c(x, y)-\bar{c}(x)$. (c) Colourmap of the horizontal velocity $u$. (d) Colourmap of the vertical velocity $v$. Note that the x-axis has been compressed by a factor of 10 in these plots.
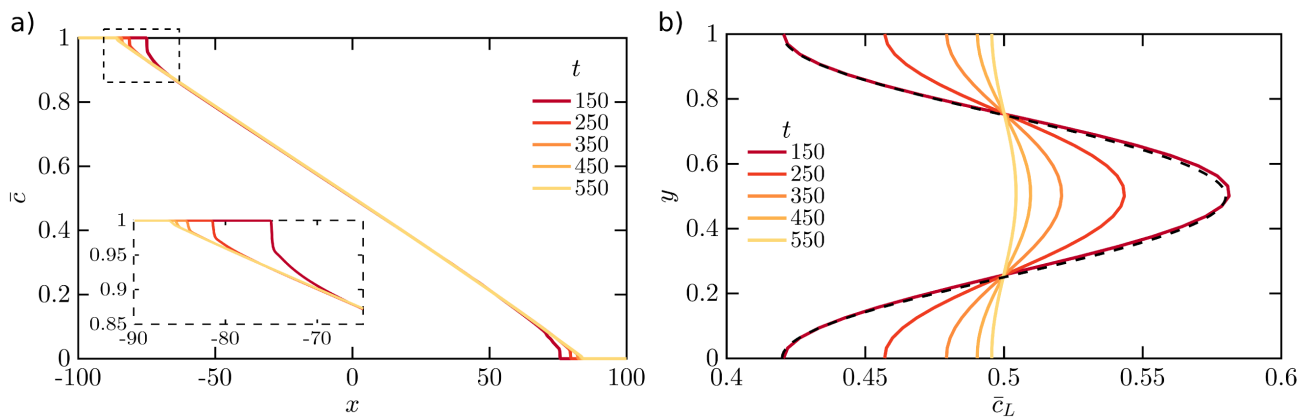

Figure 10. (a) Plot of the transversely averaged concentration. (b) Plot of the longitudinally averaged concentration $\bar{c}_{L}(y)=\int_{-\Gamma / 2}^{\Gamma / 2} c(x, y) d x=\frac{1}{2}+\int_{-\Gamma / 2}^{\Gamma / 2} c^{\prime}(x, y) d x$. A sinusoidal fit for $t=150$ is given by the dashed black line. In both plots $R=2, \mathrm{Pe}=2000$ and the time, given by the colour, ranges from 150 to 550 .

smoothly in the transverse direction. Accounting for the other approximations in the model is the subject of future work.

\section{Late times: single-finger exchange-flow regime}

\subsection{Numerical observations}

At late times, we find a new flow regime which, to leading order, involves a single pair of fingers counter-propagating through a linear background concentration gradient as shown by the snapshots in figure 9 . The concentration field is dominated by a nearly uniform background gradient in the horizontal direction with some small transverse deviations superimposed (figure 9a). The concentration deviations (figure 9b) are horizontally uniform and have a single maximum (i.e. they form a single finger). The horizontal velocity $u$ (figure 9c) tracks closely the concentration deviations while the vertical velocity $v$ (figure $9 \mathrm{~d}$ ) is only appreciable at the tips.

Figure 10 shows how the concentration field evolves over time. We find that the transversely averaged concentration, $\bar{c}(x)$, is linear and steady in the interior. The fluid 

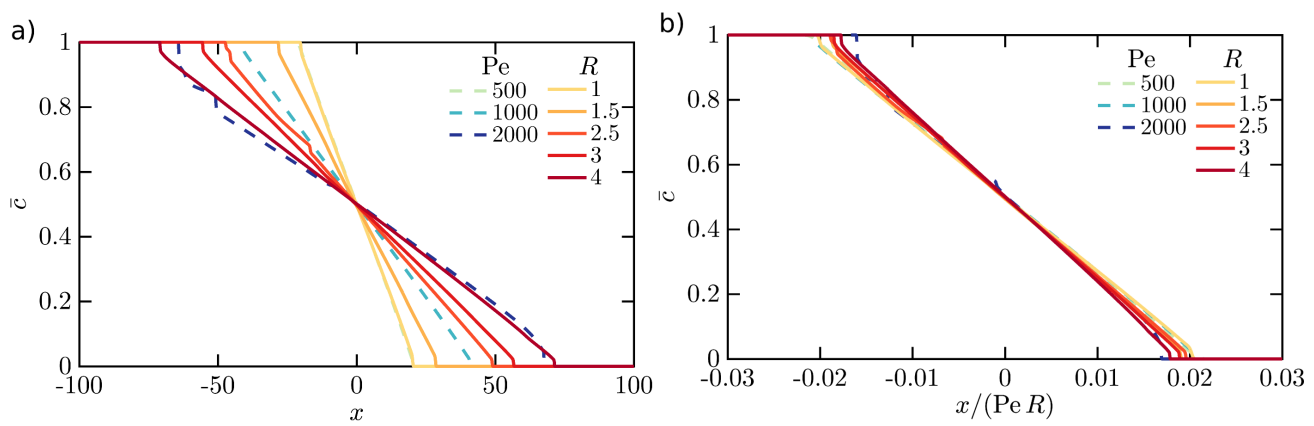

Figure 11. (a) Plots of $\bar{c}(x)$ for $R=2, \mathrm{Pe}=500,1000,2000$ (dashed) and $\mathrm{Pe}=1000$ and $R=1,1.5,2.5,3,4$ (solid) at $t=200$. (b) $\bar{c}$ as a function of $x /(R \mathrm{Pe})$, with the fitted line $\bar{c}=1 / 2-24.9 x / R P e$ (black, dashed)

flow only widens the mixing region by filling in the linear profile (inset to figure 10a). In addition, we find that $\bar{c}$ is no longer skewed and $\bar{c}=1 / 2$ is in the middle of the domain. These features are in stark contrast to the previous regime in which $\bar{c}(x)$ was asymmetric and non-linear. Superimposed on this background concentration field are horizontally uniform deviations which are sinusoidal in the transverse direction (figure 10b). These deviations decay in time, which ultimately results in a one-dimensional linear concentration field that evolves purely by diffusion in the $x$ direction.

\subsection{Asymptotic model}

The late-time regime is characterized by a linear background gradient with a single pair of counter-propagating fingers superimposed. The fingers have a very large aspect ratio, and so the velocity is given by (5.7), which for small deviations $c^{\prime}$, reduces to

$$
u=R c^{\prime}+O\left(c^{\prime 2}\right) .
$$

We look for a steady interior solution for $\bar{c}$, for which (2.18) becomes

$$
\frac{1}{\mathrm{Pe}} \frac{\partial^{2} \bar{c}}{\partial x^{2}}=O\left(c^{\prime 2}\right)
$$

or

$$
\bar{c}=-\alpha x+\frac{1}{2}+O\left(c^{\prime 2}\right) .
$$

Given that the net change in concentration of the two fluids must be equal and opposite, the concentration at the mid-plane must be $1 / 2$, which determines the constant of integration in (6.3).

Substituting the steady transversely averaged concentration (6.3) and velocity (6.1) into equation (2.19) results in a partial differential equation for the evolution of the deviations,

$$
\frac{\partial c^{\prime}}{\partial t}-\alpha R c^{\prime}=\frac{1}{\mathrm{Pe}} \frac{\partial^{2} c^{\prime}}{\partial y^{2}}+O\left(\frac{c^{\prime}}{\mathrm{Pe} h^{2}}\right)+O\left(c^{\prime 2}\right) .
$$

This equation is independent of $x$; therefore, the deviations must be horizontally uniform, as observed. The single-finger solution to the leading order truncation of (6.4) is,

$$
c^{\prime}(y, t)=\sin (2 \pi y) e^{-\gamma\left(t-t^{*}\right)},
$$



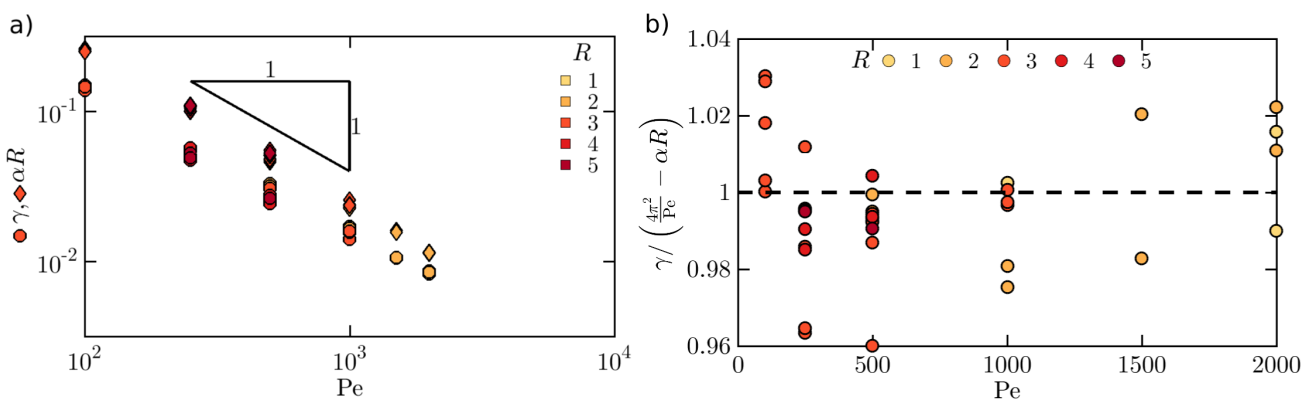

Figure 12. (a) Plot of $\gamma$ (circles) and $\alpha R$ (diamonds) as functions of Pe and $R$ (colours). (b) Ratio of $\gamma$, measured from the simulations as the decay rate of the maximum of $c^{\prime}$ at $x=0$, to $\gamma(\alpha R)$, calculated using equation (6.6) where $\alpha$ is the slope of $\bar{c}$ measured at $t>200$.

where $t^{*}$ is a virtual origin relating to the transition between regimes and

$$
\gamma=\frac{4 \pi^{2}}{\mathrm{Pe}}-\alpha R
$$

Note that, while a solution of (6.4) with any integer number of fingers is permissible, solutions with more fingers decay more rapidly over time, and the solution with $k=2 \pi$ (6.5) is the slowest decaying mode.

The slope $\alpha$ of the interior profile in (6.3) is set by the amount of mixing that occurs during the intermediate, non-linear regime. That regime lasts for a time $t \sim O(\mathrm{Pe})$ during which the mixing zone grows linearly in time, $h \sim R t$. Therefore, once the system has reached the single-finger regime, the width of the mixing zone will have become $h \approx 1 / \alpha \sim R \mathrm{Pe}$, such that $\alpha R=\hat{A} / \mathrm{Pe}$, for some constant $\hat{A}$. We fit $\hat{A}=24.9$ to the collapsed transversely averaged concentration profiles (figure 11b).

We verify this model by measuring $\alpha$ and $\gamma$ from the numerical simulations. We calculate $\alpha$ by measuring the slope of $\bar{c}$ at $x=0$ at some late time, and $\gamma$ by measuring the decay rate of the maximum of $c^{\prime}$ at the mid-plane. Plots of the numerically measured $\gamma$ and $\alpha R$ are given in figure $12 \mathrm{a}$ and both quantities exhibit the predicted $1 /$ Pe scaling. Finally, the validity of equation (6.6) is tested by plotting the ratio of $\gamma$ measured from the simulations and $\gamma(\alpha)$ calculated using equation (6.6). This quantity is plotted in figure $12 \mathrm{~b}$ and deviates by a maximum of $4 \%$ over a range of Peclet numbers and log-viscosity ratios.

\subsection{Total convective mixing}

One of the major implications of this final single-finger exchange-flow regime is that the viscous-fingering instability can only generate a finite amount of convective mixing. In figure 13a we plot the time-integral of the convective flux through the midplane,

$$
F=\left.\int_{0}^{\infty} \int_{0}^{1} u c^{\prime}\right|_{x=0} d y d t
$$

as a function of the Peclet number and log-viscosity ratio. We find that the flux increases linearly with both $\mathrm{Pe}$ and $R$ for $\mathrm{Pe} \gg 1$, and can be fit by the functional form $F=$ $\hat{a} R(\mathrm{Pe}-\hat{b} / R)$, where the shift in the Peclet number, $\hat{b} / R$, corresponds to the onset of the instability as described in section 5.1. We find that the numerical data is best fit with $\hat{a}=5.3 \times 10^{-3}$ and $\hat{b}=45$ (solid lines in figure $13 \mathrm{a}$ ).

Of course, provided advection dominates the horizontal transport, the quantity $F$ can also be directly related to the slope $\alpha$ of the late-time profiles, by mass conservation. 

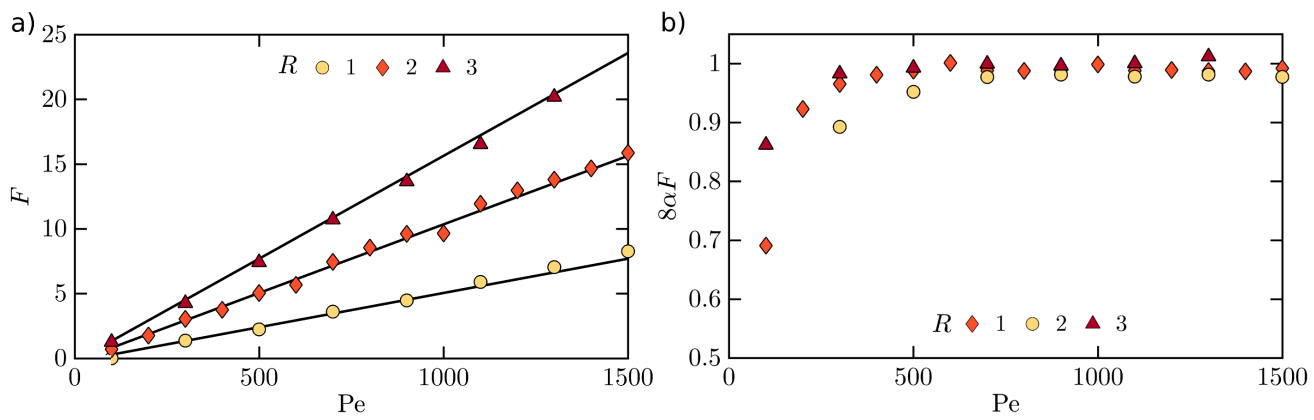

Figure 13. (a) Time-integrated convective exchange flux $F$ (6.7) between the two fluids as a function of Pe and $R$ (colours), as calculated from the numerical simulations. In order to calculate the infinite time integral in (6.7), we integrate the numerical data out to $t=200$, which is well into the late-time regime in all simulations, and fit a decaying exponential function $E(t)$ to the flux $\left.\int_{0}^{1} u c^{\prime}\right|_{x=0} d y$ for subsequent times such that $F=\left.\int_{0}^{200} \int_{0}^{1} u c^{\prime}\right|_{x=0} d y d t+\int_{200}^{\infty} E(t) d t$. The lines of best fit (black) correspond to the fit $F=5.3 \times 10^{-3} R(\mathrm{Pe}-45 / R)$. (b) Ratio of the time-integrated convective exchange flux and the final slope of the transversely averaged concentration.

Such a balance gives

$$
F=\frac{1}{8 \alpha}
$$

For the three values of $R$ plotted, we find that this prediction gives good agreement for $\mathrm{Pe}>O(100)$ (figure 13b), which suggests that for the range of $R$ plotted, horizontal diffusion plays a negligible role in mixing for $\mathrm{Pe}>O(100)$.

\section{Discussion and Conclusions}

In this paper, we have investigated miscible viscous fingering in a semi-infinite planar geometry using high-resolution simulations. We identified three distinct regimes: an earlytime linearly unstable regime, an intermediate-time non-linear regime, and a late-time single-finger exchange-flow regime. In each of these regimes, we identify the predominant balances and scalings for the mixing length $h$, the number of fingers $n$, and the total convective transport $\mathrm{Nu}$ (table 1 ).

The dimensional characteristic length scales of the flow structures, and the time scales over which they evolve, are summarized in table 2 . The early-time dynamics are set by a local balance of advection and diffusion at the finger scale and hence are independent of the width of the porous medium $a$. The flow is more unstable - that is, the flow has finer structures and faster growth rates - when the viscosity contrast and velocity are large, or the diffusivity is small. Once the instability has had enough time to diffuse transversely across the entire width of the porous medium, which occurs at a time scale $T \sim a^{2} / D$, the flow enters the late-time regime. In this case, a single pair of counter-propagating fingers remain, which occupy half of the width of the domain respectively.

In section 5.1, we showed that for sufficiently small Peclet numbers, the flow can skip the intermediate regime, and for even smaller Peclet numbers, the instability can be suppressed altogether. We then presented a linear stability analysis to identify this cut-off for the instability and compared it to numerical experiments.

In section 5.2 we attempted to improve on current models for the transversely averaged concentration in the non-linear regime. We started by deriving the simple Koval model, identified where it disagreed with the numerical simulations and improved on one of its 


\begin{tabular}{c|c} 
Early-times & Late-times \\
\hline$X \sim \phi D / R U$ & $X \sim R U a^{2} / \phi D$ \\
$Y \sim \phi D / R U$ & $Y \sim a$ \\
$T \sim \phi^{2} D / R^{2} U^{2}$ & $T \sim a^{2} / D$
\end{tabular}

TABLE 2. Dimensional length and time scales for early and late times.

shortcomings by including a simple model of the nearly parabolic concentration profile across propagating fingers. We used this ansatz to derive the effective viscosity of the fingered region (5.13), in good agreement with both the numerical simulations and the empirical fit to the Koval model.

Finally, in section 6 we identified a new single-finger exchange-flow regime in which the flow consists of a linear background gradient and counter-propagating fingers. These fingers exponentially decay and convection stops leaving a linear background gradient. We derived a model for the asymptotic behaviour and showed that it agrees with the numerical simulations. One important consequence of this eventual shutdown is that there is a maximum amount of convective mixing that the instability can generate. Since diffusion coefficients for typical pairs of fluids tend to be very small, this shutdown is most relevant when the displacement process occurs at very small scales (small $a$ ) or very long times.

To illustrate the relevant length and time scales in the late-time regime, we use parameter values from the $\mathrm{CO}_{2}$ sequestration project at Sleipner to estimate the 'shutdown time', $T_{s d}$, taken to reach the late-time regime and the 'final' mixing zone width $H$. We take the parameter values of the carbon-dioxide/brine system to be as follows (Neufeld et al. 2010; Boait et al. 2012): background velocity, which is the buoyancy velocity, $U=4 \times 10^{-6} \mathrm{~ms}^{-1}$; log-viscosity ratio $R=2.5$; porosity $\phi=0.3$; aquifer thickness $a=10 \mathrm{~m}$; and diffusivity $D=2 \times 10^{-9} \mathrm{~m}^{2} \mathrm{~s}^{-1}$. In this case, the diffusivity is taken to be the molecular diffusivity of carbon dioxide and brine $D_{m}$. Note that this is only valid when the pore-scale Peclet number, defined as $U a_{p} / D_{m}$ ( $a_{p}$ is the size of the pores), is small; otherwise the effective diffusivity is given by an anisotropic velocity-dependent dispersion tensor that could be significantly larger than $D_{m}$ (Lake 1989).

Using these parameters, the Peclet number of the flow is $\mathrm{Pe}=7 \times 10^{4}$. The time until shutdown can be approximated from the numerical simulations as $T_{s d} \approx 10^{-1} a^{2} / D$ which gives a shutdown time of approximately 150 years. Furthermore, the mixing zone can be approximated as $H=10^{-1.5} R a^{2} U / \phi D$ which gives a $50 \mathrm{~km}$ long mixing zone upon shutdown. In contrast, if the interface were stable and the mixing at the interface only occurred through diffusion, the width of the mixing zone would grow like $\sqrt{4 D t}$, which, after 150 years, would be approximately $5 \mathrm{~m}$.

In a real porous medium, some of the assumptions we made during the analysis may no longer hold. For instance, the dispersion can be anistropic and velocity dependent; the permeability often varies spatially at a variety of length scales (pore-scale to field-scale) and with varying degrees of randomness (from completely random to highly structured); and the fluids can be only partially miscible. While some of these topics have been discussed in the context of the onset problem and early-time behaviour (Zimmerman \& Homsy 1991, 1992; De Wit \& Homsy 1997a,b; Tan \& Homsy 1992; Nicolaides et al. 2015), their impact on the late-time behaviour remains to be understood. 


\section{Acknowledgements}

J.S.N is supported by a Gates Cambridge Scholarship, D.R.H is supported by a Research Fellowship at Gonville and Caius College, Cambridge and J.A.N is partly supported by a Royal Society University Research Fellowship.

\section{REFERENCES}

AdAms, J.C. 1999 Computational information systems laboratory. mudpack: Multigrid software for elliptic partial differential equations.

Almarcha, C., Trevelyan, P.M.J., Grosfils, P. \& De Wit, A. 2010 Chemically driven hydrodynamic instabilities. Phys. Rev. Lett. 104, 144501.

Bischofberger, I., Ramachandran, R. \& Nagel, S.R. 2014 Fingering versus stability in the limit of zero interfacial tension. Nat. Comm. $\mathbf{5 2 6 5}$ (5).

Blackwell, R.J., Rayne, J.R. \& Terry, W.M. 1959 Factors influencing the efficiency of miscible displacements. Petr. Trans. AIME 216, 1-8.

Boait, F.C., White, N.J., Bickle, M.J., Chadwick, R.A., Neufeld, J.A. \& Huppert, H.E. 2012 Spatial and temporal evolution of injected co2 at the sleipner field, north sea.

J. Geophys. Res. 117.

Воотн, R.J.S 2010 On the growth of the mixing zone in miscible viscous fingering. J. Fluid Mech. 655, 527-539.

Chui, J.Y.Y., De Anna, P. \& Juanes, R. 2015 Interface evolution during radial miscible viscous fingering. Phys. Rev. E $041003(\mathrm{R})$.

De Wit, A. \& Homsy, G. M. 1997 a Viscous fingering in periodically heterogeneous porous media. I. Formulation and linear instability. J. Chem. Phys. 107 (22), 9609.

De Wit, A. \& Homsy, G. M. $1997 b$ Viscous fingering in periodically heterogeneous porous media. II. Numerical simulations. J. Chem. Phys. 107 (22), 9619.

Hewitt, D.R., Neufeld, J.A. \& Lister, J. R. 2013 Convective shutdown in a porous medium at high Rayleigh number. J. Fluid Mech. 719, 551-586.

Hill, S. 1952 Channelling in packed columns. Chem. Eng. Sci. 1, 247-253.

Huppert, H.E. \& Neufeld, J.A. 2014 The fluid mechanics of carbon dioxide sequestration. Ann. Rev. Fluid Mech. 46, 255-272.

Islam, M.N. \& AzAiEz, J. 2005 Fully implicit finite difference pseudo-spectral method for simulating high mobility-ratio miscible displacements. Int. J. Num. Meth. Fluids 1, 161183.

Jha, B., Cueto-Felgueroso, L. \& Juanes, R. $2011 a$ Fluid mixing from viscous fingering. Phys. Rev. Lett. 106 (19), 194502.

Jha, B., Cueto-Felgueroso, L. \& Juanes, R. $2011 b$ Quantifying mixing in viscously unstable porous media flows. Phys. Rev. E 84, 066312.

van Keken, P.E., Hauri, E.H. \& Ballentine, C.J. 2002 Mantle Mixing: The Generation, Preservation, and Destruction of Chemical Heterogeneity. Ann. Rev. Earth Planet. Sci. 30, 493-525.

Koval, E.J. 1963 A Method for Predicting the Performance of Unstable Miscible Displacement in Heterogeneous Media. Soc. Petr. Eng. J. 3 (02), 145-154.

Lajeunesse, E., Martin, J., Rakotomalala, N., Salin, D. \& Yortsos, Y.C. 1999 Miscible displacement in a Hele-Shaw cell at high rates. J. Fluid Mech. pp. 299-319.

LAKe, L.W. 1989 Enhanced oil recovery. Prentice Hall.

LELE, S.K. 1992 Compact finite difference schemes with spectral-like resolution. J. Comp. Phys. 103 (1), 16-42.

Malhotra, S., Sharma, M. M. \& Lehman, E.R. 2015 Experimental study of the growth of mixing zone in miscible viscous fingering. Phys. Fluids 27, 014105.

McCloud, K.V. \& Maher, J.V. 1995 Experimental perturbations to Saffman-Taylor flow. Phys. Rep. 260, 139-185.

Neufeld, Jerome A., Hesse, Marc A., Riaz, Amir, Hallworth, Mark A., Tchelepi, Hamdi A. \& Huppert, Herbert E. 2010 Convective dissolution of carbon dioxide in saline aquifers. Geophys. Res. Lett. 37 (22).

Nicolaides, C., Jha, B., Cueto-Felgueroso, L. \& Juanes, R. 2015 Impact of viscous 
fingering and permeability heterogeneity on fluid mixing in porous media. Water Resour. Res. 51 (4), 2634-2647.

Pramanik, Satyajit \& Mishra, Manoranjan 2015a Effect of Peclet number on miscible rectilinear displacement in a Hele-Shaw cell. Phys. Rev. E 033006.

Pramanik, S. \& Mishra, M. $2015 b$ Nonlinear simulations of miscible viscous fingering with gradient stresses in porous media. Chem. Eng. Sci. 122, 523-532.

Ruith, M. \& Meiburg, E. 2000 Miscible rectilinear displacements with gravity override. Part 1. Homogeneous porous medium. J. Fluid Mech. 420, 225-257.

Saffman, P. G. \& Taylor, G. 1958 The Penetration of a Fluid into a Porous Medium or HeleShaw Cell Containing a More Viscous Liquid. Proc.Roy. Soc. A 245 (1242), 312-329.

Stone, H.A., Stroock, A.D. \& AJdari, A. 2004 Engineering flows in small devices: microfluidics toward a lab-on-a-chip. Ann. Rev. Fluid Mech. 36, 381-411.

TAN, C.T. \& Homsy, G.M. 1987 Stability of miscible displacements in porous media: Radial source flow. Phy. Fluids 30 (5), 1239-1245.

Tan, C.T. \& Homsy, G.M. 1992 Viscous fingering with permeability heterogeneity. Phys. Fluids 4 (6), 1099-1101.

Tan, C. T. \& Homsy, G. M. 1986 Stability of miscible displacements in porous media: Rectilinear flow. Phys. Fluids 29 (11), 3549.

Tan, C. T. \& Homsy, G. M. 1988 Simulation of nonlinear viscous fingering in miscible displacement. Phys. Fluids 31 (6), 1330.

Yortsos, Y. C. \& SAlin, D. 2006 On the selection principle for viscous fingering in porous media. J. of Fluid Mech. 557, 225.

Zhou, Q. 2013 Temporal evolution and scaling of mixing in two-dimensional Rayleigh-Taylor turbulence. Phys. Fluids 25.

Zimmerman, W. B. \& Homsy, G. M. 1991 Nonlinear viscous fingering in miscible displacement with anisotropic dispersion. Phys. Fluids 3 (8), 1859.

Zimmerman, W. B. \& Homsy, G. M. 1992 Viscous fingering in miscible displacements: Unification of effects of viscosity contrast, anisotropic dispersion, and velocity dependence of dispersion on nonlinear finger propagation. Phys. Fluids 4 (11), 2348. 